\title{
Neural Circuit Reconstruction Using Serial Block-face Scanning Microscopy (SBFSEM) in Mouse Retina
}

Wan-Qing Yu ${ }^{1}$, Chi-Chou Huang ${ }^{2}$, Hideki Sasaki ${ }^{2}$, Sean McElroy $^{2}$, Gaoyu Xiao ${ }^{2}$, James Lee ${ }^{2}$, Luciano Lucas $^{2}$ and Rachel Wong ${ }^{1}$

${ }^{1}$ University of Washington, Seattle, Washington, United States, ${ }^{2}$ DRVision Technologies LLC, Bellevue, Washington, United States

How neural circuits assemble during development is a fundamental question in neuroscience. The detailed synaptic arrangement between neurons requires imaging resolution beyond light microscopy (LM). In the past decade, emerging technological advancements in volume electron microscopy and sample preparation procedures enable high-resolution imaging of large neural tissues [1]. Along with the advances in analysis software and computing infrastructure, the annotation and visualization of neural circuits are feasible.

In this talk, I will present how we use serial block-face scanning electron microscopy (SBFSEM) [2] to understand neural circuit assembly in mouse retina. First, I will give a brief overview on SBFSEM including tissue preparation, 3D volume image acquisition and post-processing of image stacks. Then, detailed examples will be provided to discuss the routine analysis we perform currently, including skeleton tracing, 3D surface reconstruction and identification of synaptic sites. There are still many challenges along the entire process. The labor-intensive data analysis limits the scale at which neural connectivity can be elucidated. Although machine learning tools have made significant progress in automatic segmentation and neuron tracing, it often requires retraining for a new dataset. Moreover, the implementation of the algorithms is usually not easy for a non-expert. In order to overcome some of the challenges, we are developing and implementing a novel image analysis pipeline, which includes a range of deep learning tools which will perform perform faster and more reliable post-processing, automatic boundary segmentation and 3D neurite tracing in EM images. Most importantly, we are designing an integrated workflow which makes data analysis of 3D EM images more accessible and efficient. Furthermore, there is an increasing demand for correlative light and electron microscopy (CLEM). I will discuss our efforts to align LM and EM image stacks to correlate anatomical features identified under fluorescence imaging with their ultrastructures. Examples using the near-infrared branding (NIRB) [3] method will be provided to illustrate an approach using CLEM to identify connectivity of specific cell types in the mouse retina.

\section{References}

[1] Briggman, K. L., \& Bock, D. D. (2012). Volume electron microscopy for neuronal circuit reconstruction. Current opinion in neurobiology, 22(1), 154-161.

[2] Denk W, Horstmann H. Serial block-face scanning electron microscopy to reconstruct threedimensional tissue nanostructure. PLoS Biol 2004, 2:e329.

[3] Bishop D, Nikic I, Brinkoetter M, Knecht S, Potz S, Kerschensteiner M, Misgeld T. Near-infrared branding efficiently correlates light and electron microscopy. Nat Methods 2011, 8:568-570. 